$$
\frac{\partial u_{p}}{\partial r}=\frac{p u_{p}}{r}+\sum_{k=0}^{n} \beta_{k} u_{p+k}+\left(\frac{2}{r e^{i \theta}}\right) \sum_{k=0}^{m} \sum_{\nu=0}^{m+n} \frac{\alpha_{k} \gamma_{\nu} u_{p+k+\nu+1}}{2 p+2 \nu+1}
$$

if $E$ has the form II; and

$$
\begin{array}{r}
\frac{\partial u_{p}}{\partial r}=\frac{p u_{p}}{r}+\left(\frac{2}{r e^{i \theta}}\right) \sum_{k=0}^{m} \sum_{\nu=0}^{n}\left(\frac{\alpha_{k} \beta_{\nu}}{2 p+2 \nu+1}\right) u_{p+k+\nu+1} \\
+\gamma_{0} u_{p}+\sum_{k=1}^{m+n}\left(\gamma_{k}-\frac{2}{r e^{i \theta}} \gamma_{k-1}\right) u_{p+k}
\end{array}
$$

if $E$ has the form III.

University of Wisconsin at Milwaukee

\title{
ON GAUSS' AND TCHEBYCHEFF'S QUADRATURE FORMULAS
}

\section{J. GERONIMUS}

The well known Gauss' Quadrature Formula

$$
\int_{-\infty}^{\infty} G_{k}(x) d \psi(x)=\sum_{i=1}^{n} \rho_{i}^{(n)} G_{k}\left(\xi_{i}^{(n)}\right)
$$

is valid for every polynomial $G_{k}(x)$, of degree $k \leqq 2 n-1$, the $\left\{\xi_{i}^{(n)}\right\}$ being the roots of the polynomial $P_{n}(x)$, orthogonal with respect to the distribution $d \psi(x)(i=1,2, \cdots, n ; n=1,2, \cdots) .^{1}$ If the sequence $\left\{P_{n}(x)\right\}$ is that of Tchebycheff (trigonometric) polynomials, then the Christoffel numbers $\rho_{i}^{(n)}, i=1,2, \cdots, n$, are equal, and the two quadrature formulas of Gauss and Tchebycheff coincide:

$$
\int_{-\infty}^{\infty} G_{k}(x) d \psi(x)=\rho_{n} \sum_{i=1}^{n} G_{k}\left(\xi_{i}^{(n)}\right), \quad k \leqq 2 n-1 ; n=1,2, \cdots
$$

The converse-that this is the only case of coincidence of these formulas-was proved by R. P. Bailey [1a] and, under more restrictive conditions, by Krawtchouk [1b] (cf. also [2]).2

We shall give here four distinct proofs of this statement, without imposing any restrictions on $\psi(x)$.

Received by the editors June 1, 1943.

${ }^{1} \psi(x)$ is a bounded non-decreasing function, with infinitely many points of increase, for which all moments exist: $c_{n}=\int_{-\infty}^{\infty} x^{n} d \psi(x) ; n=0,1,2, \cdots$.

${ }^{2}$ Numbers in brackets refer to the bibliography at the end of the paper. 
Consider the sequence of complex numbers $\left\{c_{n}\right\}, n=0,1,2, \cdots$, subject to the conditions

$$
\Delta_{n+1} \equiv\left|c_{i+k}\right|_{i, k=0}^{n} \neq 0 ; \quad n=0,1,2, \cdots .
$$

Consider also the Stieltjes linear functional $\sigma$, with

$$
\sigma\left(x^{k}\right)=c_{k} ; \quad k=0,1,2, \cdots,
$$

and the polynomials $\left\{P_{n}(x)\right\}, n=0,1,2, \cdots$, orthogonal relative to the sequence $\left\{c_{n}\right\}$, that is $[3,4]$

$$
\sigma\left\{P_{n}(x) P_{m}(x)\right\}= \begin{cases}0, & m \neq n, \\ h_{n} \equiv \Delta_{n+1} / \Delta_{n} \neq 0, & m=n .\end{cases}
$$

Let $\left\{\xi_{i}^{(n)}\right\}, i=1,2, \cdots, n$, be the roots, distinct or not, of $P_{n}(x)$, $n=1,2, \cdots$.

I. The first method of proving our statement consists in proving the following theorem.

THEOREM. From the validity of the formula

$$
\rho_{n} \sum_{i=1}^{n}\left(\xi_{i}^{(n)}\right)^{k}=c_{k}, \quad n \geqq\left[\frac{k+1}{2}\right],
$$

for $k \leqq 2$, it follows that the $\left\{P_{n}(x)\right\}$ are the Tchebycheff polynomials, so that (6) holds for all integral $k$.

Introduce the mean of order $\nu$ of the numbers $\left\{\xi_{i}^{(n)}\right\}, i=1,2, \cdots, n$ :

$$
\mu_{\nu}^{(n)}=\left[\frac{1}{n} \sum_{i=1}^{n}\left(\xi_{i}^{(n)}\right)^{\nu}\right]^{1 / \nu}, \quad \nu=1,2, \cdots ; n=1,2, \cdots .
$$

On equating the coefficients of $x^{n-1}$ and $x^{n-2}$ on both sides of the recurrence relation

$$
\begin{aligned}
P_{n}(x)=\left(x-\alpha_{n}\right) P_{n-1}(x)-\lambda_{n} P_{n-2}(x), \\
\lambda_{n} \neq 0, \quad P_{-1} \equiv 0, n=1,2, \cdots,
\end{aligned}
$$

we get

$$
\delta_{1}^{(n)}=-\sum_{k=1}^{n} \alpha_{k}, \quad \delta_{2}^{(n)}=-\sum_{k=2}^{n} \lambda_{k}+\sum_{r=2}^{n} \alpha_{r} \sum_{k=1}^{r-1} \alpha_{k},
$$

where we let

$$
P_{n}(x)=x^{n}+\delta_{1}^{(n)} x^{n-1}+\delta_{2}^{(n)} x^{n-2}+\cdots+\delta_{n}^{(n)} .
$$


On putting $k=0,1,2$ in (6) we obtain

$$
\rho_{n}=c_{0} / n ; \quad \mu_{1}^{(n)}=c_{1} / c_{0}, n \geqq 1 ; \quad \mu_{2}^{(n)}=c_{2} / c_{0}, n \geqq 2 .
$$

Since, by (9) and $\left(9^{\prime}\right)$,

$$
\begin{aligned}
n \mu_{1}^{(n)} & =-\delta_{1}^{(n)}=\sum_{k=1}^{n} \alpha_{k}, \\
n\left(\mu_{2}^{(n)}\right)^{2} & =\left(\delta_{1}^{(n)}\right)^{2}-2 \delta_{2}^{(n)}=\left(\sum_{k=1}^{n} \alpha_{k}\right)^{2}+2 \sum_{k=1}^{n} \lambda_{k}-2 \sum_{r=2}^{n} \sum_{k=1}^{r-1} \alpha_{k},
\end{aligned}
$$

we find:

$$
\alpha_{1}=\left(\alpha_{1}+\alpha_{2}\right) / 2=\left(\alpha_{1}+\alpha_{2}+\alpha_{3}\right) / 3=\cdots,
$$

whence we have the fundamental result:

$$
\alpha_{1}=\alpha_{2}=\cdots=\alpha,
$$

and similarly,

$$
\lambda_{3}=\lambda_{4}=\cdots=\lambda_{2} / 2=\lambda .
$$

Here $\alpha$ and $\lambda$ are independent of $n$. The solution of (8) under these conditions is

$$
\begin{aligned}
P_{n}(x)= & {\left[\left(x-\alpha+\left((x-\alpha)^{2}-4 \lambda\right)^{1 / 2}\right)^{n}\right.} \\
& \left.+\left(x-\alpha-\left((x-\alpha)^{2}-4 \lambda\right)^{1 / 2}\right)^{n}\right] / 2^{n}, \quad n=1,2, \cdots,
\end{aligned}
$$

which shows that the $\left\{P_{n}(x)\right\}$ are the Tchebycheff polynomials, whence the validity of (6) for $k=3,4, \cdots$ follows.

We have found incidentally the following property of the Tchebycheff polynomials.

COROLlaRY. If the arithmetic mean and the root-mean-square of the roots $\left\{\xi_{i}^{(n)}\right\}, i=1,2, \cdots, n$, of the orthogonal polynomials $P_{n}(x)$, $n=1,2, \cdots$, do not depend on $n$, the means of all orders $\nu \leqq 2 n-1$ possess the same property, and the $\left\{P_{n}(x)\right\}$ are the Tchebycheff polynomials (13).

II. Define

$$
R_{n-1}(y)=\sigma\left\{\left(P_{n}(y)-P_{n}(x)\right) /(y-x)\right\},
$$

a polynomial of degree $n-1 ; n=1,2, \cdots$. Our second proof consists in showing that (6) implies

$$
R_{n-1}(y) \equiv\left(c_{0} / n\right) P_{n}^{\prime}(y), \quad n \geqq 2 .
$$


From (6), wherein $\rho_{n}$ necessarily equals $c_{0} / n$, we get, by virtue of (4),

$$
\sigma\left(x^{k}\right)=\frac{c_{0}}{n} \sum_{i=1}^{n}\left(\xi_{i}^{(n)}\right)^{k} ; \quad \sigma\left(G_{k}(x)\right)=\frac{c_{0}}{n} \sum_{i=1}^{n} G_{k}\left(\xi_{i}^{(n)}\right), \quad n \geqq\left[\frac{k+1}{2}\right] .
$$

The desired relation

$R_{n-1}(y)=\frac{c_{0}}{n} \sum_{i=1}^{n} \frac{P_{n}(y)-P_{n}\left(\xi_{i}^{(n)}\right)}{y-\xi_{i}^{(n)}}=\frac{c_{0}}{n} P_{n}(y) \sum_{i=1}^{n} \frac{1}{y-\xi_{i}^{(n)}} \equiv \frac{c_{0}}{n} P_{n}^{\prime}(y)$

follows. Now there are different methods of showing that (14) implies (13).

$\mathrm{II}_{1}$. This statement is proved in a note [2] as a particular case of more general theorems.

$\mathrm{II}_{2}$. Introduce the polynomials $\left\{P_{n}^{(i)}(x)\right\}, i=1,2, \cdots, n$-the denominators of the convergents of order $n$ of the continued fractions ${ }^{3}$

$$
\frac{\lambda_{i} \mid}{\mid x-\alpha_{i}}-\frac{\lambda_{i+1} \mid}{\mid x-\alpha_{i+1}}-\cdots, \quad i=1,2, \cdots .
$$

We have $P_{n}^{(1)}(x) \equiv P_{n}(x) ; P_{n}^{(2)}(x) \equiv\left(1 / c_{0}\right) R_{n}(x), n=0,1,2, \cdots$.

It is easy to show that

$$
P_{n}(x)=\left(x-\alpha_{1}\right) P_{n-1}^{(2)}(x)-\lambda_{2} P_{n-2}^{(3)}(x), \quad n=2,3, \cdots .
$$

On the other hand

$$
R_{n-1}(x)=\left(x-\alpha_{n}\right) R_{n-2}(x)-\lambda_{n} R_{n-3}(x), \quad n=3,4, \cdots .
$$

Using (8), (14) and (17), we find that

$$
P_{n-1}(x)=\left(x-\alpha_{n}\right) P_{n-2}^{(2)}(x)-2 \lambda_{n} P_{n-3}^{(2)}(x), \quad n=3,4, \cdots,
$$

which gives, in conjunction with (16),

$$
\left(\alpha_{1}-\alpha_{n}\right) P_{n-2}^{(2)}(x)=2 \lambda_{n} P_{n-3}^{(2)}(x)-\lambda_{2} P_{n-3}^{(3)}(x), \quad n=3,4, \cdots .
$$

Hence

$$
\begin{aligned}
\alpha_{3}=\alpha_{4}=\cdots= & \alpha_{1}=\alpha ; \quad \lambda_{3}=\lambda_{4}=\cdots=\lambda_{2} / 2=\lambda ; \\
& P_{n-3}^{(2)}(x) \equiv P_{n-3}^{(3)}(x) .
\end{aligned}
$$

This identity, for $n=4$, gives $\alpha_{2}=\alpha$, and thus we have arrived again at $\left(12,12^{\prime}\right)$.

$\mathrm{II}_{3}$. On putting

They were introduced by Stieltjes [5]; cf. also Perron [6]. 


$$
R_{n}(y)=c_{0}\left(y^{n}+d_{1}^{(n)} y^{n-1}+\cdots+d_{n}^{(n)}\right),
$$

we find from (17) the relations

$$
d_{1}^{(n-1)}=-\sum_{k=2}^{n} \alpha_{k}, \quad d_{2}^{(n-1)}=\sum_{k=3}^{n} \alpha_{k} \sum_{r=2}^{k-1} \alpha_{r}-\sum_{k=3}^{n} \lambda_{k}
$$

analogous to (9). From (14) we find

$$
n d_{1}^{(n-1)}=(n-1) \delta_{1}^{(n)} \text {, }
$$

whence again $\alpha_{1}=\alpha_{2}=\cdots=\alpha$; and in the same way, the condition

$$
n d_{2}^{(n-1)}=(n-2) \delta_{2}^{(n)}
$$

again implies $\lambda_{3}=\lambda_{4}=\cdots=\lambda_{2} / 2=\lambda$.

We have found incidentally the following property of the Tchebycheff polynomials.

COROLlaRY. If the three highest coefficients of the polynomials $\left\{n R_{n-1}(x)\right\}$ and $\left\{c_{0} P_{n}^{\prime}(x)\right\}, n=1,2, \cdots$, coincide, then these polynomials are identical, and the $\left\{P_{n}(x)\right\}$ are the Tchebycheff polynomials (13).

\section{BIBLIOGRAPHY}

1. a. R. P. Bailey, Convergence of sequences of linear operators, Duke Math. J. vol. 2 (1936) pp. 285-303. b. M. Krawtchouk, Sur une question algébrique dans le problème des moments, Journal de l'Institut mathématique, Académie des sciences d'Ukraine, vol. 2 (1934) pp. 87-92.

2. J. Besikovitch, Calculus of finite differences (in Russian), Leningrad, 1939.

3. J. Geronimus, On polynomials orthogonal with regard to a given sequence of numbers and on Hahn's theorem, Bull. Acad. Sci. URSS. vol. 4 (1940) pp. 215-228.

4. - On polynomials orthogonal with regard to a given sequence of numbers, Transactions of the Kharkoff Mathematical Society vol. 17 (1940) pp. 3-18.

5. T. Stieltjes, Recherches sur les fractions continues, Oeuvres, vol. 2, pp. 402-566.

6. O. Perron, Die Lehre von den Kettenbriichen, Leipzig, 1939.

KHARKoff State University 